\title{
Isoperimetric Inequalities for Infinite Hyperplane Systems
}

\author{
R. Schneider
}

Mathematisches Institut, Albert-Ludwigs-Universität, Albertstrasse 23b, D-79104 Freiburg i. Br., Germany rschnei@sun1.ruf.uni-freiburg.de

\begin{abstract}
Let $\mathscr{S}$ be an infinite discrete system of $k$-dimensional flats in $n$-dimensional Euclidean space. If the total $k$-dimensional volume of the flats in $\mathscr{S}$ intersected with the ball of center 0 and radius $r$, divided by the volume of that ball, tends to a limit for $r \rightarrow \infty$, then this limit is called the density of $\mathscr{S}$. We consider isoperimetric problems of the following kind. If $\mathscr{S}$ is a hyperplane system of positive density, find sharp upper bounds for the density of the system of $k$-flats $(k \in\{0, \ldots, n-2\})$ that are generated as intersections of hyperplanes in $\mathscr{S}$. Ideas from the theory of uniform distribution of sequences are employed to define a large class of hyperplane systems, called uniform, for which all necessary densities exist, isperimetric inequalities can be proved, and systems with extremal intersection densities can be characterized.
\end{abstract}

\section{Introduction}

With an infinite discrete set $\mathscr{P}$ of points in the plane $\mathbb{R}^{2}$ it may be possible to associate a density by means of the definition

$$
D(\mathscr{P}):=\lim _{R \rightarrow \infty} \frac{\operatorname{card}(\mathscr{P} \cap R K)}{\lambda_{2}(R K)},
$$

where $K \subset \mathbb{R}^{2}$ is some compact convex set with the origin as an interior point, and $\lambda_{2}$ denotes the Lebesgue measure in $\mathbb{R}^{2}$. In cases of geometric interest, this density usually exists and is independent of the choice of $K$.

If $\mathscr{L}$ is an infinite discrete set of lines in $\mathbb{R}^{2}$, it seems natural to define a density of $\mathscr{L}$ by

$$
D_{1}(\mathscr{L}):=\lim _{R \rightarrow \infty} \frac{\sum_{L \in \mathscr{L}} \lambda_{1}(L \cap R K)}{\lambda_{2}(R K)}
$$


provided this limit exists and is independent of $K$; here $\lambda_{1}$ denotes the onedimensional Lebesgue measure. For a system $\mathscr{L}$ of lines we may consider the set $\mathscr{P}_{\mathscr{L}}$ of all intersection points of lines in $\mathscr{L}$ and then define $D_{0}(\mathscr{L}):=D\left(\mathscr{P}_{\mathscr{L}}\right)$, if this density exists.

In this paper we are interested in problems of the following kind: find a sharp upper bound for the intersection point density $D_{0}(\mathscr{L})$, given the density $D_{1}(\mathscr{L})$ of the line system $\mathscr{L}$. (The only general lower bound, of course, is zero.)

We first consider a very simple example, namely, a line system consisting of finitely many lattices of parallel lines. More precisely, let unit vectors $u_{1}, \ldots, u_{m} \in \mathbb{R}^{2}$ and positive numbers $a_{1}, \ldots, a_{m}$ be given, where $m \geq 2$, and let

$$
\mathscr{L}:=\bigcup_{i=1}^{m}\left\{L_{i}+k a_{i} u_{i}: k \in \mathbb{Z}\right\}
$$

where $L_{i}$ is a line orthogonal to $u_{i}(i=1, \ldots, m)$. In this case it is easy to see that the densities $D_{1}(\mathscr{L})$ and $D_{0}(\mathscr{L})$ exist and that $D_{1}(\mathscr{L})=\sum_{i=1}^{m} a_{i}^{-1}$. It can be shown that

$$
D_{0}(\mathscr{L}) \leq \frac{1}{2 m}\left(\cot \frac{\pi}{2 m}\right) D_{1}(\mathscr{L})^{2}
$$

and hence

$$
D_{0}(\mathscr{L}) \leq \frac{1}{\pi} D_{1}(\mathscr{L})^{2}
$$

(see (34) below). The equality sign in (4) holds if and only if $\pm u_{1}, \ldots, \pm u_{m}$ are the outer normal vectors of the edges of a regular $2 m$-gon and $a_{1}=\cdots=a_{m}$.

Definition (2) is not the only conceivable analogue of (1). Instead of measuring the density of a line system $\mathscr{L}$ by the total length of the intersections of the lines in $\mathscr{L}$ with a large convex domain $R K$, one might also think of measuring it by the total number of the lines meeting $R K$, in each case divided by a suitable power of $R$. In other words, we propose to study also the density defined by

$$
\Delta_{1}(\mathscr{L}, K):=\lim _{R \rightarrow \infty} \frac{\operatorname{card}\{L \in \mathscr{L}: L \cap R K \neq \varnothing\}}{2 R}
$$

provided the limit exists. This density depends not only on the size of $K$ but also on its shape. One may then ask for those shapes $K$ for which large homothets "catch" many intersection points while intersecting relatively few lines. If we normalize $K$ so that it has area one, then for line systems $\mathscr{L}$ of type (3) the sharp inequality

$$
D_{0}(\mathscr{L}) \leq \Delta_{1}(\mathscr{L}, K)^{2} \quad\left[\lambda_{2}(K)=1\right]
$$

holds (see (36), together with (27)). Equality in (7) holds precisely if $K$ is a centrally symmetric polygon with normal vectors $\pm u_{1}, \ldots, \pm u_{m}$ such that the edge corresponding to $u_{i}$ has length $\alpha / a_{i}$ and $\alpha$ is chosen so that $\lambda_{2}(K)=1$. 
In the following we obtain sharp estimates of types (5) and (7) for infinite hyperplane systems in $n$-dimensional space $\mathbb{R}^{n}$. Our main concern, however, is to find suitable conditions on infinite hyperplane arrangements that, on one hand, imply the existence of various densities and enable us to treat isoperimetric problems for them, and, on the other hand, are sufficiently general to admit fairly irregular systems of hyperplanes. One of the incentives for this is inequality (5): clearly this inequality cannot be improved for line systems of type (3) with unrestricted $m$. However, for no line system of type (3) is equality attained in (5). The question arises naturally whether a class of line systems, including those of type (3), exists for which the densities $D_{1}$ and $D_{0}$ exist and are nonzero, the inequality (5) holds, and the equality sign is attained by suitable systems. An affirmative answer is given below, in a more general context. We apply some ideas from the theory of uniformly distributed sequences to define what we call uniform hyperplane systems. For these, some sharp inequalities of isoperimetric type for intersection densities are obtained. The final techniques, for proving such inequalities, have been used before in stochastic geometry, in connection with stationary Poisson hyperplane processes. Thus the principal aim of this paper is to be seen in the introduction of the uniform hyperplane systems, which permit us to transfer some ideas from probabilistic geometry to the more intuitive field of discrete geometry.

\section{Uniform Hyperplane Systems}

We work in $n$-dimensional real Euclidean vector space $\mathbb{R}^{n}(n \geq 2)$, with scalar product $\langle\cdot, \cdot\rangle$. By $\lambda_{k}$ we denote the $k$-dimensional Lebesgue measure in $k$ dimensional affine subspaces of $\mathbb{R}^{n}$. For the Lebesgue measure in $\mathbb{R}$ we use the notation $\lambda$. The set $B^{n}:=\left\{x \in \mathbb{R}^{n}:\langle x, x\rangle \leq 1\right\}$ is the unit ball of $\mathbb{R}^{n}$ and $S^{n-1}:=$ $\left\{x \in \mathbb{R}^{n}:\langle x, x\rangle=1\right\}$ is the unit sphere. By $\kappa_{k}$ we denote the volume of the $k$ dimensional unit ball. By a measure on $S^{n-1}$ we always understand a finite Borel measure on $S^{n-1}$. The unique rotation invariant probability measure on $S^{n-1}$ (normalized spherical Lebesgue measure) is denoted by $\sigma$.

Every hyperplane $H \subset \mathbb{R}^{n}$ has a representation

$$
H=H_{u, t}=\left\{x \in \mathbb{R}^{n}:\langle x, u\rangle=t\right\}
$$

with $u \in S^{n-1}$ and $t \in \mathbb{R}$. If $0 \notin H$, we can assume that $t>0$, and then this representation is unique. In this case, $u$ is the unit normal vector of $H$ pointing into the half-space bounded by $H$ that does not contain 0 , and $t$ is the distance of $H$ from the origin.

We consider an infinite system $\mathscr{H}$ of hyperplanes in $\mathbb{R}^{n}$ with the property that every ball in $\mathbb{R}^{n}$ meets only finitely many hyperplanes of $\mathscr{H}$. We choose the origin 0 so that $0 \notin H$ for $H \in \mathscr{H}$ and that no two hyperplanes of $\mathscr{H}$ are at the same distance from 0 . Then we can enumerate the hyperplanes of $\mathscr{H}$ such that $\mathscr{H}=\left\{H_{u_{i}, t_{i}}\right\}_{i \in \mathbb{N}}$ with $t_{i}<t_{i+1}$ for $i \in \mathbb{N}$.

In order to be able to define and apply various densities connected with $\mathscr{H}$, we impose on $\mathscr{H}$ a property that is motivated by the theory of uniformly distributed sequences (see, e.g., [4]). We briefly recall the underlying notions. 
First let $\omega=\left(x_{i}\right)_{i \in \mathbb{N}}$ be a sequence in the interval $[0,1]$. For $0 \leq \alpha<\beta \leq 1$ and for $N \in \mathbb{N}$, let

$$
A(\omega,[\alpha, \beta), N):=\operatorname{card}\left\{i \in \mathbb{N}: x_{i} \in[\alpha, \beta), i \leq N\right\} .
$$

The sequence $\left(x_{i}\right)_{i \in \mathbb{N}}$ is said to be uniformly alistributed in $[0,1]$ if

$$
\lim _{N \rightarrow \infty} \frac{A(\omega,[\alpha, \beta), N)}{N}=\beta-\alpha
$$

for $0 \leq \alpha<\beta \leq 1$. An equivalent condition is that

$$
\lim _{N \rightarrow \infty} \frac{1}{N} \sum_{i=1}^{N} f\left(x_{i}\right)=\int_{0}^{1} f d \lambda
$$

for every continuous function $f:[0,1] \rightarrow \mathbb{R}$.

Property (9) has been generalized as follows. Let $X$ be a compact Hausdorff space and let $\varphi \neq 0$ be a finite regular Borel measure on $X$. The sequence $\left(x_{i}\right)_{i \in \mathbb{N}}$ in $X$ is called $\varphi$-uniformly distributed if

$$
\lim _{N \rightarrow \infty} \frac{1}{N} \sum_{i=1}^{N} f\left(x_{i}\right)=\frac{1}{\varphi(X)} \int_{X} f d \varphi
$$

for every continuous function $f: X \rightarrow \mathbb{R}$.

For a sequence $\omega=\left(x_{i}\right)_{i \in \mathbb{N}}$ in $X$ and for $M \subset X, N \in \mathbb{N}$ let

$$
A(\omega, M, N):=\operatorname{card}\left\{i \in \mathbb{N}: x_{i} \in M, i \leq N\right\} .
$$

A Borel set $M \subset X$ is called a $\varphi$-continuity set if $\varphi(\partial M)=0$; here $\partial M$ denotes the boundary of $M$. The sequence $\left(x_{i}\right)_{i \in \mathbb{N}}$ is $\varphi$-uniformly distributed in $X$ if and only if

$$
\lim _{N \rightarrow \infty} \frac{A(\omega, M, N)}{N}=\frac{\varphi(M)}{\varphi(X)}
$$

for all $\varphi$-continuity sets $M \subset X[4$, p. 175].

For our definition of a uniform hyperplane system $\mathscr{H}$ we now combine two conditions in a suitable way: the condition that the normal vectors of the hyperplanes in $\mathscr{H}$ should be uniformly distributed in $S^{n-1}$, in the sense of (11) with some measure $\varphi$, and a variant of (8) for the distances of the hyperplanes in $\mathscr{H}$ from the origin.

Let the hyperplane system $\mathscr{H}=\left\{H_{u_{i}, i_{i}}\right\}_{i \in \mathbb{N}}$ be given. For a subset $B \subset S^{n-1}$ and for numbers $0 \leq \alpha<\beta \leq 1$ and $R>0$, let

$$
A(\mathscr{H}, B,[\alpha, \beta), R):=\operatorname{card}\left\{i \in \mathbb{N}: u_{i} \in B, R \alpha \leq t_{i}<R \beta\right\} .
$$

Definition. The hyperplane system $\mathscr{H}$ is said to be $\varphi$-uniform, where $\varphi \neq 0$ is a finite measure on the sphere $S^{n-1}$, if

$$
\lim _{R \rightarrow \infty} \frac{A(\mathscr{H}, B,[\alpha, \beta), R)}{R}=\varphi(B)(\beta-\alpha)
$$


for every $\varphi$-continuity set $B \subset S^{n-1}$ and for $0 \leq \alpha<\beta \leq 1 . \mathscr{H}$ is called uniform if it is $\varphi$-uniform for some finite measure $\varphi$.

Suppose that the hyperplane system $\mathscr{H}$ is $\varphi$-uniform. Define

$$
N_{R}:=\operatorname{card}\left\{i \in \mathbb{N}: t_{i}<R\right\} \quad \text { for } \quad R>0 .
$$

From (12) with $B=S^{n-1}$ and $\alpha=0, \beta=1$ we get

$$
\lim _{R \rightarrow \infty} \frac{N_{R}}{R}=\varphi\left(S^{n-1}\right) .
$$

For any $\varphi$-continuity set $B \subset S^{n-1}$ we deduce that

$$
\begin{aligned}
\lim _{N \rightarrow \infty} \frac{A\left(\left(u_{i}\right)_{i \in \mathbb{N}}, B, N\right)}{N} & =\lim _{R \rightarrow \infty} \frac{A\left(\left(u_{i}\right)_{i \in \mathbb{N}}, B, N_{R}\right)}{N_{R}} \\
& =\lim _{R \rightarrow \infty} \frac{R}{N_{R}} \frac{A(\mathscr{H}, B,[0,1), R)}{R}=\frac{\varphi(B)}{\varphi\left(S^{n-1}\right)} .
\end{aligned}
$$

Thus the sequence $\left(u_{i}\right)_{i \in \mathbb{N}}$ of normal vectors of $\mathscr{Z}$ is $\varphi$-uniformly distributed in $S^{n-1}$. It should, however, be observed that condition (12) is considerably stronger: it involves some product or independence structure for the directions of the hyperplanes and their distances from the origin.

Simple examples of uniform hyperplane systems can be constructed by extending (3). More generally, let $\varphi$ be a measure on $S^{n-1}$ that is concentrated on a set $\left\{u_{i}\right\}_{i \in \mathbb{N}^{\prime}}$, where $\mathbb{N}^{\prime}=\{1, \ldots, m\}$ or $\mathbb{N}^{\prime}=\mathbb{N}$, and let $a_{i}^{-1}:=\varphi\left(\left\{u_{i}\right\}\right)>0$. Then

$$
\mathscr{B}:=\bigcup_{i \in \mathbb{N}^{\prime}}\left\{H_{u_{i}, k a_{i}}: k=1,2,3, \ldots\right\}
$$

is a $\varphi$-uniform hyperplane system. (Observe also that $a_{i} \rightarrow \infty$ for $i \rightarrow \infty$, so that every ball in $\mathbb{R}^{n}$ meets only finitely many hyperplanes of $\mathscr{H}$.) The existence of $\varphi$-uniform hyperplane systems for any given finite measure $\varphi \neq 0$ on $S^{n-1}$ is proved in Theorem 2.2.

In the following we use the abbreviation $S^{n-1} \times[0,1]=: Z$. For any compact topological space $X$, we write $C(X)$ for the Banach space of real continuous functions on $X$ with the supremum norm, denoted by $\|\cdot\|$. The indicator function of a subset $M \subset X$ is denoted by $\mathbf{1}_{M}$ (the domain $X$ will be clear from the context).

Theorem 2.1. Let $\varphi \neq 0$ be a finite measure on $S^{n-1}$. The hyperplane system $\mathscr{H}=\left\{H_{u_{i}, t_{1}}\right\}_{i \in \mathbb{N}}$ is $\varphi$-uniform if and only if it satisfies

$$
\lim _{R \rightarrow \infty} \frac{1}{R} \sum_{\substack{i \in \mathbb{N} \\ t_{i}<R}} f\left(u_{i}, \frac{t_{i}}{R}\right)=\int_{Z} f d \varphi \otimes \lambda
$$

for every function $f \in C(Z)$. 
Proof. Assume that (14) is satisfied. Let $M \subset Z$ be a $\varphi \otimes \lambda$-continuity set. It can be proved as on p. 175 of [4] (observe that $\varphi$ is regular, by Proposition 7.2.3 in [2]) that

$$
\lim _{R \rightarrow \infty} \frac{1}{R} \sum_{\substack{i \in \mathbb{N} \\ t_{i}<R}} \mathbf{1}_{M}\left(u_{i}, \frac{t_{i}}{R}\right)=\varphi \otimes \lambda(M)
$$

Let $B \subset S^{n-1}$ be a $\varphi$-continuity set and let $0 \leq \alpha<\beta \leq 1$. Applying (15) to $M:=B \times[\alpha, \beta)$, we obtain

$$
\lim _{R \rightarrow \infty} \frac{1}{R} \operatorname{card}\left\{i \in \mathbb{N}: u_{i} \in B, \alpha \leq \frac{t_{i}}{R}<\beta\right\}=\varphi(B)(\beta-\alpha)
$$

which is (12). Thus the system $\mathscr{H}$ is $\varphi$-uniform.

Conversely, suppose that $\mathscr{Z}$ is $\varphi$-uniform. Let $f \in C(Z)$. First we assume that $f$ is of the form $f(u, t)=g(u) h(t)$ with $g \in C\left(S^{n-1}\right)$ and $h \in C([0,1])$. Let $0<\varepsilon<1$ be given. As on p. 176 of [4] it can be shown that there are $\varphi$-continuity sets $B_{1}, \ldots, B_{k} \subset S^{n-1}$ and numbers $\gamma_{1}, \ldots, \gamma_{k}$ such that the function

$$
g_{\varepsilon}:=\sum_{r=1}^{k} \gamma_{r} \mathbf{1}_{B_{r}}
$$

defined on $S^{n-1}$ satisfies $\left\|g-g_{\varepsilon}\right\| \leq \varepsilon$. Similarly, there are intervals $I_{r}=\left[\alpha_{r}, \beta_{r}\right) \subset$ $[0,1], r=1, \ldots, m$, and numbers $\eta_{1}, \ldots, \eta_{m}$ such that the function

$$
h_{\varepsilon}:=\sum_{r=1}^{m} \eta_{r} \mathbf{1}_{I_{r}}
$$

defined on $[0,1]$ satisfies $\left\|h-h_{\varepsilon}\right\| \leq \varepsilon$. We conclude that $\left\|f-g_{\varepsilon} h_{\varepsilon}\right\| \leq c \varepsilon$ with $c=\|g\|+\|h\|+1$. By (12),

$$
\lim _{R \rightarrow \infty} \frac{1}{R} \sum_{\substack{i \in \mathbb{N} \\ t_{i}<R}} \mathbf{1}_{B_{r}}\left(u_{i}\right) \mathbf{1}_{I_{s}}\left(\frac{t_{i}}{R}\right)=\varphi\left(B_{r}\right) \lambda\left(I_{s}\right)=\int_{Z} \mathbf{1}_{B_{r}} \mathbf{1}_{I_{s}} d \varphi \otimes \lambda
$$

for $r=1, \ldots, k$ and $s=1, \ldots, m$. By linearity, it follows that

$$
\lim _{R \rightarrow \infty} \frac{1}{R} \sum_{\substack{i \in \mathbb{N} \\ t_{i}<R}} g_{\varepsilon}\left(u_{i}\right) h_{\varepsilon}\left(\frac{t_{i}}{R}\right)=\int_{Z} g_{\varepsilon} h_{\varepsilon} d \varphi \otimes \lambda
$$


Similarly, as on p. 173 of [4], we deduce that

$$
\begin{aligned}
& \left|\frac{1}{R} \sum_{\substack{i \in \mathbb{N} \\
t_{i}<R}} f\left(u_{i}, \frac{t_{i}}{R}\right)-\int_{Z} f d \varphi \otimes \lambda\right| \\
& \leq \frac{1}{R} \sum_{\substack{i \in \mathbb{N} \\
t_{i}<R}}\left|\left(f-g_{\varepsilon} h_{\varepsilon}\right)\left(u_{i}, \frac{t_{i}}{R}\right)\right|+\int_{Z}\left|f-g_{\varepsilon} h_{\varepsilon}\right| d \varphi \otimes \lambda \\
& +\left|\frac{1}{R} \sum_{\substack{i \in \mathbb{N} \\
t_{i}<R}} g_{\varepsilon}\left(u_{i}\right) h_{\varepsilon}\left(\frac{t_{i}}{R}\right)-\int_{Z} g_{\varepsilon} h_{\varepsilon} d \varphi \otimes \lambda\right| \\
& \leq c \varepsilon \frac{N_{R}}{R}+c \varepsilon \varphi\left(S^{n-1}\right)+\left|\frac{1}{R} \sum_{\substack{i \in \mathbb{N} \\
t_{i}<R}} g_{\varepsilon}\left(u_{i}\right) h_{\varepsilon}\left(\frac{t_{i}}{R}\right)-\int_{Z} g_{\varepsilon} h_{\varepsilon} d \varphi \otimes \lambda\right| .
\end{aligned}
$$

By (13) and (16), this is less than $3 c \varphi\left(S^{n-1}\right) \varepsilon$ if $R$ is sufficiently large. Thus (14) holds for this special function $f$.

If now $f \in C(Z)$ is arbitrary, we can approximate $f$ uniformly by functions of the form

$$
(u, t) \mapsto \sum_{j=1}^{m} g_{j}(u) h_{j}(t)
$$

with $m \in \mathbb{N}, g_{j} \in C\left(S^{n-1}\right)$, and $h_{j} \in C([0,1])$, as follows from the Stone-Weierstraß theorem. We deduce that (14) holds for $f$, which completes the proof.

Let $\varphi \neq 0$ be a finite measure on $S^{n-1}$ and suppose that the hyperplane system $\left\{H_{u_{i}, t_{i}}\right\}_{i \in \mathbb{N}}$ is $\varphi$-uniform. If $c>0$, then the system $\left\{H_{u_{i}, c t_{i}}\right\}_{i \in \mathbb{N}}$ is $(\varphi / c)$-uniform. Hence, if we want to show the existence of $\varphi$-uniform hyperplane systems, we may assume, without loss of generality, that $\varphi$ is a probability measure.

In order to prove the existence of many $\varphi$-uniform hyperplane systems, we use a random construction. Let $\varphi$ be a probability measure on $S^{n-1}$. We choose an infinite sequence $\left(u_{1}, u_{2}, \ldots\right)$ of independent random unit vectors, each with probability distribution $\varphi$. With $u_{i}$ we associate the hyperplane $H_{u_{i}, i}$, which has unit normal vector $u_{i}$ and distance $i$ from the origin. Theorem 2.2 below shows that the resulting hyperplane system $\left\{H_{u_{i},}\right\}_{i \in \mathbb{N}}$ is almost surely $\varphi$-uniform.

We consider the product probability measure $P:=\otimes_{i=1}^{\infty} \varphi$ on the product space $X:=\prod_{i=1}^{\infty} S^{n-1}$. With each element $\omega=\left(u_{i}\right)_{i \in \mathbb{N}} \in X$ we associate the hyperplane system $\mathscr{H}(\omega):=\left\{H_{u_{i}, i}\right\}_{i \in \mathbb{N}^{*}}$. The proof of the following theorem is an adaptation of the proof of Theorem 2.2 on p. 183 of [4].

Theorem 2.2. Let $S$ be the set of all sequences $\omega \in X$ for which the system $\mathscr{X}(\omega)$ is $\varphi$-uniform. Then $P(S)=1$. 
Proof. First let $f \in C\left(S^{n-1}\right)$ be a function satisfying $\int_{S^{n-1}} f d \varphi=0$. Let $0 \leq \alpha<\beta$ $\leq 1$. For $N \in \mathbb{N}$, define the function $F_{N}$ on $X$ by

$$
F_{N}\left(u_{1}, u_{2}, \ldots\right):=\frac{1}{N} \sum_{N \alpha \leq i<N \beta} f\left(u_{i}\right)
$$

As on p. 182 of [4] it can be shown that

$$
\int_{X} F_{N}^{2} d P=\frac{\operatorname{card}\{i \in \mathbb{N}: N \alpha \leq i<N \beta\}}{N^{2}} \int_{S^{n-1}} f^{2} d \varphi \leq \frac{1}{N} \int_{S^{n-i}} f^{2} d \varphi
$$

and deduced that $\lim _{m \rightarrow \infty} F_{m^{2}}=0 P$-a.e. If $N \in \mathbb{N}$, then $m^{2} \leq N<(m+1)^{2}$ for suitable $m \in \mathbb{N}$, and

$$
F_{N}\left(u_{1}, u_{2}, \ldots\right)=\frac{1}{N} \sum_{m^{2} \alpha \leq i<m^{2} \beta} f\left(u_{i}\right)-\frac{1}{N} \sum_{m^{2} \alpha \leq i<N \alpha} f\left(u_{i}\right)+\frac{1}{N} \sum_{m^{2} \beta \leq i<N \beta} f\left(u_{i}\right) .
$$

It follows that

$$
\left|F_{N}\right| \leq\left|F_{m^{2}}\right|+\frac{4 m+2}{m^{2}}\|f\|
$$

and hence $\lim _{N \rightarrow \infty} F_{N}=0 P$-a.e.

If $f \in C\left(S^{n-1}\right)$ is arbitrary, then the foregoing applies to $f-c$ with $c:=\int_{S^{n-1}} f$ $d \varphi$. Since

$$
\frac{1}{N} \sum_{N \alpha \leq i<N \beta} 1=\beta-\alpha+\frac{\delta}{N} \quad \text { with } \quad|\delta| \leq 1,
$$

we deduce that

$$
\lim _{N \rightarrow \infty} \frac{1}{N} \sum_{N \alpha \leq i<N \beta} f\left(u_{i}\right)=(\beta-\alpha) \int_{S^{n-1}} f d \varphi
$$

for $P$-almost all sequences $\left(u_{1}, u_{2}, \ldots\right) \in X$.

Now let $\mathscr{F}$ be a countable dense subset of $C\left(S^{n-1}\right)$. Then for $P$-almost all sequences $\left(u_{1}, u_{2}, \ldots\right) \in X$ it is true that (17) holds for each $f \in \mathscr{F}$ and for arbitrary rational numbers $\alpha, \beta$ satisfying $0 \leq \alpha<\beta \leq 1$. Let $\left(u_{1}, u_{2}, \ldots\right) \in X$ be such a sequence, and consider the hyperplane system $\mathscr{H}=\left\{H_{u_{i}, t_{1}}\right\}_{i \in \mathbb{N}}$ with $t_{i}=i$ for $i \in \mathbb{N}$.

Let $f \in C(Z)$, and assume first that $f(u, t)=g(u) h(t)$ with $g \in C\left(S^{n-1}\right)$ and $h \in C([0,1])$. For given $0<\varepsilon<1$, there is a function $g_{\varepsilon} \in \mathscr{F}$ for which $\left\|g-g_{\varepsilon}\right\|<\varepsilon$, and there are rational intervals $\left[\alpha_{r}, \beta_{r}\right) \subset[0,1], r=1, \ldots, m$, and numbers 
$\eta_{1}, \ldots, \eta_{m}$ such that the function

$$
h_{\varepsilon}:=\sum_{r=1}^{m} \eta_{r} \mathbf{1}_{\left[\alpha_{r}, \beta_{r}\right)}
$$

defined on $[0,1]$ satisfies $\left\|h-h_{\varepsilon}\right\| \leq \varepsilon$. For rational $\alpha, \beta$ with $0 \leq \alpha<\beta \leq 1$ we have

$$
\begin{aligned}
\sum_{\substack{i \in \mathbb{N} \\
t_{i}<R}} g_{\varepsilon}\left(u_{i}\right) \mathbf{1}_{[\alpha, \beta)}\left(\frac{t_{i}}{R}\right) \\
\quad=\sum_{R \alpha \leq i<R \beta} g_{\varepsilon}\left(u_{i}\right) \\
\quad=\sum_{[R] \alpha \leq i<[R] \beta} g_{\varepsilon}\left(u_{i}\right)-\sum_{[R] \alpha \leq i<R \alpha} g_{\varepsilon}\left(u_{i}\right)+\sum_{[R] \beta \leq i<R \beta} g_{\varepsilon}\left(u_{i}\right),
\end{aligned}
$$

where $[R]$ denotes the integer part of $R$. Each of the last two sums has at most one summand. It follows that

$$
\begin{aligned}
\lim _{R \rightarrow \infty} \frac{1}{R} \sum_{\substack{i \in \mathbb{N} \\
i_{i}<R}} g_{\varepsilon}\left(u_{i}\right) \mathbf{1}_{[\alpha, \beta)}\left(\frac{t_{i}}{R}\right) & =\lim _{N \rightarrow \infty} \frac{1}{N} \sum_{N \alpha \leq i<N \beta} g_{\varepsilon}\left(u_{i}\right) \\
& =(\beta-\alpha) \int_{S^{n-1}} g_{\varepsilon} d \varphi=\int_{Z} g_{\varepsilon} \mathbf{1}_{(\alpha, \beta)} d \varphi \otimes \lambda .
\end{aligned}
$$

By linearity, we conclude that

$$
\lim _{R \rightarrow \infty} \frac{1}{R} \sum_{\substack{i \in \mathbb{N} \\ t_{l}<R}} g_{\varepsilon}\left(u_{i}\right) h_{\varepsilon}\left(\frac{t_{i}}{R}\right)=\int_{Z} g_{\varepsilon} h_{\varepsilon} d \varphi \otimes \lambda
$$

As in the proof of Theorem 2.1 (after (16)) we deduce that (14) holds for arbitrary functions $f \in C(Z)$. By Theorem 2.1, the hyperplane system $\mathscr{K}$ is $\varphi$-uniform. This completes the proof.

We conclude this section with two technical remarks which are needed later.

For $k \in \mathbb{N}$, let $Z^{k}=\prod_{r=1}^{k} Z$ and $(\varphi \otimes \lambda)^{k}=\otimes_{r=1}^{k}(\varphi \otimes \lambda)$. Suppose that $\mathscr{H}=\left\{H_{u_{i}, t_{i}}\right\}_{i \in \mathbb{N}}$ is a $\varphi$-uniform hyperplane system and that $f \in C\left(Z^{k}\right)$. Then

$$
\lim _{R \rightarrow \infty} \frac{1}{R^{k}} \sum_{\substack{i_{1} \in \mathbb{N} \\ t_{i_{1}}<R}} \cdots \sum_{\substack{i_{k} \in \mathbb{N} \\ t_{i_{k}}<R}} f\left(u_{i_{1}}, \frac{t_{i_{1}}}{R}, \ldots, u_{i_{k}}, \frac{t_{i_{k}}}{R}\right)=\int_{Z^{k}} f d(\varphi \otimes \lambda)^{k} .
$$


For the proof, we assume first that

$$
f\left(u_{1}, t_{1}, \ldots, u_{k}, t_{k}\right)=g_{1}\left(u_{1}, t_{1}\right) \cdots g_{k}\left(u_{k}, t_{k}\right)
$$

with $g_{r} \in C(Z)$ for $r=1, \ldots, k$. Then

$$
\frac{1}{R^{k}} \sum_{\substack{i_{1} \in \mathbb{N} \\ t_{i_{1}}<R}} \cdots \sum_{\substack{i_{k} \in \mathbb{N} \\ t_{t_{k}}<R}} f\left(u_{i_{1}}, \frac{t_{i_{1}}}{R}, \ldots, u_{i_{k}}, \frac{t_{i_{k}}}{R}\right)=\prod_{r=1}^{k} \frac{1}{R} \sum_{\substack{i_{r} \in \mathbb{N} \\ t_{t_{r}}<R}} g_{r}\left(u_{i_{r}}, \frac{t_{i_{r}}}{R}\right) .
$$

For $R \rightarrow \infty$, this tends to

$$
\begin{aligned}
& \prod_{r=1}^{k} \int_{Z} g_{r}\left(u_{r}, t_{r}\right) d(\varphi \otimes \lambda)\left(u_{r}, t_{r}\right) \\
& \quad=\int_{Z} \cdots \int_{Z} \prod_{r=1}^{k} g_{r}\left(u_{r}, t_{r}\right) d(\varphi \otimes \lambda)\left(u_{1}, t_{1}\right) \cdots d(\varphi \otimes \lambda)\left(u_{k}, t_{k}\right) \\
& \quad=\int_{Z^{k}} f d(\varphi \otimes \lambda)^{k} .
\end{aligned}
$$

Thus (18) holds for this special function $f$. Since, by the Stone-Weierstraß theorem, finite sums of such special functions are dense in $C\left(Z^{k}\right),(18)$ follows for arbitrary $f \in C\left(Z^{k}\right)$.

Our second remark concerns an extension of (18) to certain noncontinuous functions. Let $f: Z^{k} \rightarrow \mathbb{R}$ be a function with the following property:

(*) A $(\varphi \otimes \lambda)^{k}$-continuity set $M \subset Z^{k}$ exists such that $f$ is continuous in the interior of $M$ and is zero in the interior of its complement.

If $(*)$ holds, continuous functions $g_{1}, g_{2}: Z^{k} \rightarrow \mathbb{R}$ can be found, for any $\varepsilon>0$, such that $g_{1} \leq f \leq g_{2}$ and $\int_{Z^{k}}\left(g_{2}-g_{1}\right) d(\varphi \otimes \lambda)^{k} \leq \varepsilon$, and then it can be concluded that (18) holds for $f$. The necessary arguments are similar to those on pp. 175 and 3 of [4].

\section{Densities}

From now on we assume that $\mathscr{H}=\left\{H_{u_{i}, t_{t}}\right\}_{i \in \mathbb{N}}$ is a $\varphi$-uniform hyperplane system in $\mathbb{R}^{n}$, where $\varphi \neq 0$ is an even finite measure on $S^{n-1}$. A measure $\varphi$ on $S^{n-1}$ is called even if it is invariant under reflection of $S^{n-1}$ in its center. The assumption that $\varphi$ is even appears natural for the following considerations.

The hyperplanes in $\mathscr{H}$ generate lower-dimensional flats, by intersection. We want to define densities for these intersections.

Let $K \subset \mathbb{R}^{n}$ be a convex body with interior points. Since later considerations involve only the homothety class of $K$, we may assume that $K \subset B^{n}$. 
Let $k \in\{1, \ldots, n\}$. For $R>0$, we define

$$
\mu_{n-k}(\mathscr{H}, K, R):=\frac{1}{k !} \sum_{H_{1} \in \mathscr{K}} \cdots \sum_{H_{k} \in \mathscr{H}} \lambda_{n-k}^{*}\left(H_{1} \cap \cdots \cap H_{k} \cap R K\right)
$$

with

$$
\lambda_{n-k}^{*}(A):= \begin{cases}\lambda_{n-k}(A) & \text { if } \operatorname{dim} A=n-k, \\ 0 & \text { otherwise. }\end{cases}
$$

Thus $\mu_{n-k}(\mathscr{H}, K, R)$ is the sum of the $(n-k)$-dimensional volumes of the intersections of $R K$ with all $(n-k)$-flats generated as intersections of any $k$ hyperplanes in $\mathscr{H}$. If an $(n-k)$-flat is generated by different $k$-tuples in $\mathscr{H}$, it is counted with the corresponding multiplicity. We define the $(n-k)$-dimensional volume density of the hyperplane system $\mathscr{Z}$ by

$$
D_{n-k}(\mathscr{H}):=\lim _{R \rightarrow \infty} \frac{\mu_{n-k}(\mathscr{H}, K, R)}{\lambda_{n}(R K)} .
$$

That the limit exists and does not depend on $K$, is seen as follows. By definition,

$$
\begin{aligned}
k ! \frac{\mu_{n-k}(\mathscr{H}, K, R)}{\lambda_{n}(R K)} & =\frac{1}{R^{n} \lambda_{n}(K)} R^{n-k} \sum_{H_{1} \in \mathscr{H}} \cdots \sum_{H_{k} \in \mathscr{H}} \lambda_{n-k}^{*}\left(\frac{1}{R} H_{1} \cap \cdots \cap \frac{1}{R} H_{k} \cap K\right) \\
& =\frac{1}{\lambda_{n}(K)} \frac{1}{R^{k}} \sum_{\substack{i_{1} \in \mathbb{N} \\
t_{i_{1}} \leq R}} \cdots \sum_{\substack{i_{k} \in \mathbb{N} \\
t_{i_{k}} \leq R}} f\left(u_{i_{1}}, \frac{t_{i_{1}}}{R}, \ldots, u_{i_{k}}, \frac{t_{i_{k}}}{R}\right)
\end{aligned}
$$

with

$$
f\left(u_{1}, \tau_{1}, \ldots, u_{k}, \tau_{k}\right):=\lambda_{n-k}^{*}\left(H_{u_{1}, \tau_{1}} \cap \cdots \cap H_{u_{k}, \tau_{k}} \cap K\right)
$$

(Recall that $K \subset B^{n}$, so that $H_{u, \tau} \cap K \neq \varnothing$ implies $\tau \leq 1$.) The function $f$ satisfies condition $(*)$ at the end of Section 2 , e.g., with

$$
\begin{gathered}
M=:\left\{\left(u_{1}, \tau_{1}, \ldots, u_{k}, \tau_{k}\right) \in Z^{k}: \operatorname{dim} H_{u_{1}, \tau_{1}} \cap \cdots \cap H_{u_{k}, \tau_{k}}=n-k\right. \\
\left.\quad \text { and } H_{u_{1}, \tau_{1}} \cap \cdots \cap H_{u_{k}, \tau_{k}} \cap \text { int } K \neq \varnothing\right\} .
\end{gathered}
$$

Thus (18) holds for $f$. This gives

$$
\begin{gathered}
k ! \lambda_{n}(K) \lim _{R \rightarrow \infty} \frac{\mu_{n-k}(\mathscr{H}, K, R)}{\lambda_{n}(R K)} \\
=\int_{Z^{k}} f d(\varphi \otimes \lambda)^{k}
\end{gathered}
$$




$$
\begin{aligned}
& =\int_{S^{n-1}} \cdots \int_{S^{n-1}} \int_{0}^{1} \cdots \int_{0}^{1} \lambda_{n-k}^{*}\left(H_{u_{1}, \tau_{1}} \cap \cdots \cap H_{u_{k}, \tau_{k}} \cap K\right) \\
& \quad \times d \lambda\left(\tau_{1}\right) \cdots d \lambda\left(\tau_{k}\right) d \varphi\left(u_{1}\right) \cdots d \varphi\left(u_{k}\right) \\
& =2^{-k} \int_{S^{n-1}} \cdots \int_{S^{n-1}} \int_{-1}^{1} \cdots \int_{-1}^{1} \lambda_{n-k}^{*}\left(H_{u_{1}, \tau_{1}} \cap \cdots \cap H_{u_{k}, \tau_{k}} \cap K\right) \\
& \quad \times d \lambda\left(\tau_{1}\right) \cdots d \lambda\left(\tau_{k}\right) d \varphi\left(u_{1}\right) \cdots d \varphi\left(u_{k}\right),
\end{aligned}
$$

because the measure $\varphi$ is even. If $u_{1}, \ldots, u_{k} \in S^{n-1}$ are linearly independent, then

$$
\begin{aligned}
& \int_{-1}^{1} \cdots \int_{-1}^{1} \lambda_{n-k}^{*}\left(H_{u_{1}, \tau_{1}} \cap \cdots \cap H_{u_{k}, \tau_{k}} \cap K\right) d \lambda\left(\tau_{1}\right) \cdots d \lambda\left(\tau_{k}\right) \\
& \quad=\lambda_{n}(K)\left[u_{1}, \ldots, u_{k}\right]
\end{aligned}
$$

where $\left[u_{1}, \ldots, u_{k}\right]$ denotes the $k$-dimensional volume of the parallelepiped spanned by $\left[u_{1}, \ldots, u_{k}\right]$. If $u_{1}, \ldots, u_{k}$ are linearly dependent, then (20) still holds, since both sides are zero. Thus we get

$$
D_{n-k}(\mathscr{Z})=\frac{1}{2^{k} k !} \int_{S^{n-1}} \cdots \int_{S^{n-1}}\left[u_{1}, \ldots, u_{k}\right] d \varphi\left(u_{1}\right) \cdots d \varphi\left(u_{k}\right)
$$

In a similar way, we define number densities instead of volume densities. These, however, depend on the convex body $K$ used in their definition. The body $K$ need not have interior points now, but we still assume that $\operatorname{dim} K \geq 1$ and $K \subset B^{n}$. For $R>0$, let

$$
\nu_{n-k}(\mathscr{H}, K, R):=\frac{1}{k !} \sum_{H_{1} \in \mathscr{H}} \cdots \sum_{H_{k} \in \mathscr{L}} \chi_{n-k}\left(H_{1} \cap \cdots \cap H_{k} \cap R K\right)
$$

with

$$
\chi_{n-k}(A):= \begin{cases}\chi(A) & \text { if } \operatorname{dim} A=n-k \\ 0 & \text { otherwise }\end{cases}
$$

where $\chi$ denotes the Euler characteristic. Thus $\nu_{n-k}(\mathscr{H}, K, R)$ is the number of $(n-k)$-flats generated by $\mathscr{H}$ that meet $R K$. Again an $(n-k)$-flat has to be counted with the correct multiplicity if it is generated in more than one way as an intersection of $k$ hyperplanes in $\mathscr{H}$.

We define the $(n-k)$-dimensional number density, relative to $K$, of the hyperplane system $\mathscr{H}$ by

$$
\Delta_{n-k}(\mathscr{H}, K):=\lim _{R \rightarrow \infty} \frac{\nu_{n-k}(\mathscr{H}, K, R)}{\kappa_{k} R^{k}}
$$


(The factor $\kappa_{k}$ in the denominator appears for convenience.) By definition,

$$
\begin{aligned}
k ! \nu_{n-k}(\mathscr{H}, K, R) & =\sum_{H_{1} \in \mathscr{H}} \cdots \sum_{H_{k} \in \mathscr{H}} \chi_{n-k}\left(\frac{1}{R} H_{1} \cap \cdots \cap \frac{1}{R} H_{k} \cap K\right) \\
& =\sum_{\substack{i_{1} \in \mathbb{N} \\
t_{i_{1}} \leq R}} \cdots \sum_{\substack{i_{k} \in \mathbb{N} \\
t_{i_{k}} \leq R}} g\left(u_{i_{1}}, \frac{t_{i_{1}}}{R}, \ldots, u_{i_{k}}, \frac{t_{i_{k}}}{R}\right)
\end{aligned}
$$

with

$$
g\left(u_{1}, \tau_{1}, \ldots, u_{k}, \tau_{k}\right):=\chi_{n-k}\left(H_{u_{1}, \tau_{1}} \cap \cdots \cap H_{u_{k}, \tau_{k}} \cap K\right)
$$

As before, we get

$$
\begin{aligned}
k ! \lim _{R \rightarrow \infty} & \frac{\nu_{n-k}(\mathscr{H}, K, R)}{R^{k}} \\
= & \int_{Z^{k}} g d(\varphi \otimes \lambda)^{k} \\
= & 2^{-k} \int_{S^{n-1}} \cdots \int_{S^{n-1}} \int_{-1}^{1} \cdots \int_{-1}^{1} \chi_{n-k}\left(H_{u_{1}, \tau_{1}} \cap \cdots \cap H_{u_{k}, \tau_{k}} \cap K\right) \\
& \quad \times d \lambda\left(\tau_{1}\right) \cdots d \lambda\left(\tau_{k}\right) d \varphi\left(u_{1}\right) \cdots d \varphi\left(u_{k}\right) .
\end{aligned}
$$

If $u_{1}, \ldots, u_{k} \in S^{n-1}$ are linearly independent, then

$$
\begin{aligned}
& \int_{-1}^{1} \cdots \int_{-1}^{1} \chi_{n-k}\left(H_{u_{1}, \tau_{1}} \cap \cdots \cap H_{u_{k}, \tau_{k}} \cap K\right) d \lambda\left(\tau_{1}\right) \cdots d \lambda\left(\tau_{k}\right) \\
& \quad=\lambda_{k}\left(\Pi_{u_{1}, \ldots, u_{k}} K\right)\left[u_{1}, \ldots, u_{k}\right]
\end{aligned}
$$

where $\Pi_{u_{1}, \ldots, u_{k}}$ denotes the orthogonal projection from $\mathbb{R}^{n}$ onto the linear subspace spanned by $u_{1}, \ldots, u_{k}$. If $u_{1}, \ldots, u_{k}$ are linearly dependent, both sides of (23) are zero. Thus we get

$$
\begin{aligned}
\Delta_{n-k}(\mathscr{H}, K)= & \frac{1}{2^{k} k ! \kappa_{k}} \int_{S^{n-1}} \cdots \int_{S^{n-1}} \lambda_{k}\left(\Pi_{u_{1}, \ldots, u_{k}} K\right) \\
& \times\left[u_{1}, \ldots, u_{k}\right] d \varphi\left(u_{1}\right) \cdots d \varphi\left(u_{k}\right) .
\end{aligned}
$$

Especially,

$$
\Delta_{n-1}(\mathscr{H}, K)=\frac{1}{4} \int_{S^{n-1}} \lambda\left(\Pi_{u} K\right) d \varphi(u)
$$


If $h(K, \cdot)$ denotes the support function of $K$, then $\int h(K, u) d \varphi(u)=$ $\int h(K,-u) d \varphi(u)$, since $\varphi$ is even, and $h(K, u)+h(K,-u)=\lambda\left(\Pi_{u} K\right)$. Hence, we can also write

$$
\Delta_{n-1}(\mathscr{H}, K)=\frac{1}{2} \int_{S^{n-1}} h(K, u) d \varphi(u) .
$$

In special cases, the number densities $\Delta_{n-k}$ essentially reduce to volume densities. This is true for $k=n$, since

$$
\kappa_{n} \Delta_{0}(\mathscr{H}, K)=\lambda_{n}(K) D_{0}(\mathscr{H}),
$$

and for $K=B^{n}$, since

$$
\Delta_{n-k}\left(\mathscr{H}, B^{n}\right)=D_{n-k}(\mathscr{X})
$$

by (21) and (24). From (13), (21), and (28) we see, in particular, that

$$
\lim _{R \rightarrow \infty} \frac{N_{R}}{2 R}=\frac{1}{2} \varphi\left(S^{n-1}\right)=D_{n-1}(\mathscr{H})=\Delta_{n-1}\left(\mathscr{H}, B^{n}\right) .
$$

It is appropriate to call this number simply the density of $\mathscr{H}$.

\section{Inequalities for Densities}

We now establish some inequalities of isoperimetric type for the densities introduced in the previous section. We assume that $\mathscr{H}$ is a $\varphi$-uniform hyperplane system in $\mathbb{R}^{n}$, where $\varphi \neq 0$ is an even finite measure on $S^{n-1}$. If the measure $\varphi$ is concentrated on a great subsphere of $S^{n-1}$, the system $\mathscr{K}$ is called degenerate. In this case the densities are determined by the hyperplanes of $\mathscr{Z}$ that are parallel to a certain fixed line, so that the situation essentially reduces to one in a lower-dimensional space. We, therefore, assume in the following that $\mathscr{H}$ is nondegenerate. The hyperplane system $\mathscr{H}$ is called isotropic if the measure $\varphi$ is rotation invariant and thus a multiple of $\sigma$. by

With the measure $\varphi$ we associate the zonoid $Z_{\varphi}$ whose support function is given

$$
h\left(Z_{\varphi}, u\right)=\frac{1}{2} \int_{S^{n-1}}|\langle u, v\rangle| d \varphi(v) \quad \text { for } \quad u \in \mathbb{R}^{n}
$$

The intuitive interpretation of $h\left(Z_{\varphi}, u\right)$ is clear from (26): if $K_{u}$ denotes the closed segment with endpoints $u$ and $-u$, where $u \in S^{n-1}$, then $K_{u}$ has the support function $h\left(K_{u}, \cdot\right)=|\langle u, \cdot\rangle|$, hence

$$
h\left(Z_{\varphi}, u\right)=\Delta_{n-1}\left(\mathscr{H}, K_{u}\right)=\lim _{R \rightarrow \infty} \frac{1}{2 R} \operatorname{card}\left\{H \in \mathscr{H}: H \cap R K_{u} \neq \varnothing\right\} .
$$


Thus $h\left(Z_{\varphi}, u\right)$, for $u \in S^{n-1}$, can be considered as the density of the intersection points of $\mathscr{H}$ with a line in direction $u$.

Since $\varphi$ is not concentrated on a great subsphere of $S^{n-1}$, it follows from (30) that $Z_{\varphi}$ has interior points.

From (21) and a formula for zonoids (see, e.g., Theorem 5.3.3 of [9]), we get

$$
D_{n-k}(\mathscr{H})=\frac{\left(\begin{array}{l}
n \\
k
\end{array}\right)}{2^{k} \kappa_{n-k}} W_{n-k}\left(Z_{\varphi}\right) .
$$

Here $W_{0}, W_{1}, \ldots, W_{n}$ denote the quermassintegrals or Minkowski functionals (e.g., p. 290 of [9]). It is well known that

$$
W_{n-j}\left(Z_{\varphi}\right)^{k} \geq \kappa_{n}^{k-j} W_{n-k}^{j}\left(Z_{\varphi}\right)
$$

for $0<j<k \leq n$, with equality if and only if $Z_{\varphi}$ is a ball [9, pp. 334-335]. The zonoid $Z_{\varphi}$ is a ball if and only if $\varphi=c \sigma$ with $c>0$ (as follows from the uniqueness part of Theorem 3.5.3 in [9]). Thus we have obtained the following result (in stochastic geometry, a similar argument was used in [7] and [11]):

Theorem 4.1. The volume densities of any nondegenerate uniform hyperplane system $\mathscr{H}$ in $\mathbb{R}^{n}$ satisfy

$$
\left[\frac{2^{j} \kappa_{n-j}}{\left(\begin{array}{l}
n \\
j
\end{array}\right)} D_{n-j}(\mathscr{H})\right]^{k} \geq \kappa_{n}^{k-j}\left[\frac{2^{k} \kappa_{n-k}}{\left(\begin{array}{l}
n \\
k
\end{array}\right)} D_{n-k}(\mathscr{H})\right]^{j}
$$

for $0<j<k \leq n$. Equality for one pair $(j, k)$ holds if and only if $\mathscr{Z}$ is isotropic.

The particular case $j=1$ gives the sharp estimate

$$
D_{n-k}(\mathscr{H}) \leq \frac{1}{n^{k}}\left(\begin{array}{l}
n \\
k
\end{array}\right) \frac{\kappa_{n-1}^{k}}{\kappa_{n-k} \kappa_{n}^{k-1}} D_{n-1}(\mathscr{H})^{k} .
$$

Thus among all uniform hyperplane systems of given density, precisely the isotropic ones have maximal $(n-k)$-dimensional intersection density, for $k \in\{2, \ldots, n\}$.

In the case of the plane $\mathbb{R}^{2}$, formulae (31) reduce to

$$
D_{1}(\mathscr{H})=\frac{1}{4} L\left(Z_{\varphi}\right), \quad D_{0}(\mathscr{H})=\frac{1}{4} A\left(Z_{\varphi}\right),
$$

where $L$ and $A$ denote, respectively, the perimeter and area of convex domains. Inequality (33) now reads

$$
D_{0}(\mathscr{H}) \leq \frac{1}{\pi} D_{1}(\mathscr{H})^{2} .
$$


In the special case where the measure $\varphi$ is concentrated in $m$ pairs of antipodal points of $S^{1}$, the zonoid $Z_{\varphi}$ is a polygon with $2 m$ edges. An improvement of the planar isoperimetric inequality for convex polygons with a given number of edges (see p. 9 of [3]) gives

$$
D_{0}(\mathscr{H}) \leq \frac{1}{2 m}\left(\cot \frac{\pi}{2 m}\right) D_{1}(\mathscr{H})^{2}
$$

which is inequality (4). Equality holds if and only if $Z_{\varphi}$ is a regular polygon.

Inequalities can also be obtained for superpositions (i.e., unions) of uniform hyperplane systems. Let $\mathscr{Z}_{i}$ be a $\varphi_{i}$-uniform hyperplane system $(i=1,2)$. Then $\mathscr{H}_{1} \cup \mathscr{H}_{2}$ is $\varphi$-uniform with $\varphi=\varphi_{1}+\varphi_{2}$, hence

$$
D_{n-1}\left(\mathscr{H}_{1} \cup \mathscr{H}_{2}\right)=D_{n-1}\left(\mathscr{H}_{1}\right)+D_{n-1}\left(\mathscr{H}_{2}\right) \text {. }
$$

Since $Z_{\varphi}=Z_{\varphi_{1}}+Z_{\varphi_{2}}$, we see from (31) that

$$
D_{n-k}\left(\mathscr{H}_{1} \cup \mathscr{Z}_{2}\right)=\frac{\left(\begin{array}{l}
n \\
k
\end{array}\right)}{2^{k} \kappa_{n-k}} V(\underbrace{Z_{\varphi_{1}}+Z_{\varphi_{2}}, \ldots, Z_{\varphi_{1}}+Z_{\varphi_{2}}}_{k}, \underbrace{B^{n}, \ldots, B^{n}}_{n-k}),
$$

where $V(\cdot, \ldots, \cdot)$ is the mixed volume in $\mathbb{R}^{n}$. The general Brunn-Minkowski theorem (see Theorems 6.4.3 and 6.6.9 of [9]) now shows that

$$
D_{n-k}\left(\mathscr{H}_{1} \cup \mathscr{H}_{2}\right)^{1 / k} \geq D_{n-k}\left(\mathscr{H}_{1}\right)^{1 / k}+D_{n-k}\left(\mathscr{H}_{2}\right)^{1 / k}
$$

for $k=2, \ldots, n$. Equality for a number $k$ holds if and only if $\varphi_{1}$ and $\varphi_{2}$ are proportional. (For analogous results in stochastic geometry, see [11] and [5].)

Now we turn to the number densities $\Delta_{n-k}(\mathscr{H}, K)$. They depend, for $k<n$, in an essential way on the convex body $K$; hence inequalities of isoperimetric type for these densities also have to involve $K$.

First we assume that the uniform hyperplane system $\mathscr{H}$ is isotropic, that is, $c \sigma$-uniform with some $c>0$. Then

$$
\begin{aligned}
\Delta_{n-k}(\mathscr{H}, K)= & \frac{c^{k}}{2^{k} k ! \kappa_{k}} \int_{S^{n-1}} \cdots \int_{S^{n-1}} \int_{-1}^{1} \cdots \int_{-1}^{1} \chi\left(H_{u_{1}, \tau_{1}} \cap \cdots \cap H_{u_{k}, \tau_{k}} \cap K\right) \\
& \times d \lambda\left(\tau_{1}\right) \cdots d \lambda\left(\tau_{k}\right) d \sigma\left(u_{1}\right) \cdots d \sigma\left(u_{k}\right)
\end{aligned}
$$

since $\operatorname{dim} H_{u_{1}, \tau_{1}} \cap \cdots \cap H_{u_{k}, \tau_{k}}=n-k$ for $\sigma^{k}$-almost all $\left(u_{1}, \ldots, u_{k}\right)$. The multiple integral can be computed by successively applying Crofton's formula from integral geometry (see, e.g., p. 78, together with (1.9) and (2.4), [10]). The result is

$$
\Delta_{n-k}(\mathscr{H}, K)=a_{n k} c^{k} W_{n-k}(K)
$$

with a constant $a_{n k}$. Choosing $K=B^{n}$ and using $W_{n-k}\left(B^{n}\right)=\kappa_{n}$, equality (28), the 
equality case of (33), and finally (29), we see that

$$
a_{n k}=\frac{1}{(2 n)^{k}}\left(\begin{array}{l}
n \\
k
\end{array}\right) \frac{\kappa_{n-1}^{k}}{\kappa_{n-k} \kappa_{n}^{k}} .
$$

From inequality (32), with $Z_{\varphi}$ replaced by $K$, we now obtain the following result (for similar results in stochastic geometry, see p. 231 of [6] and [8]):

Theorem 4.2. Let $K \subset \mathbb{R}^{n}$ be a convex body with interior points. The number densities, relative to $K$, of an isotropic uniform hyperplane system $\mathscr{H}$ in $\mathbb{R}^{n}$ satisfy

$$
\Delta_{n-j}(\mathscr{H}, K)^{k} \geq \kappa_{n}^{k-j} \frac{a_{n j}^{k}}{a_{n k}^{j}} \Delta_{n-k}(\mathscr{H}, K)^{j}
$$

for $0<j<k \leq n$. Equality for one pair $(j, k)$ holds if and only if $K$ is a ball.

For general uniform hyperplane systems, it seems difficult to obtain information on the number density $\Delta_{n-k}(\mathscr{H}, K)$ from its integral representation (24), except for $k=n$. Following [7], we introduce the convex body $M_{\varphi}$ (determined up to a translation) with surface area measure $\varphi$. This body exists by Minkowski's theorem (e.g., Theorem 7.1.2 of [9]), since the measure $\varphi$ is even and not concentrated on a great subsphere. By (26) (and a special case of formula (5.1.18) in [9]) we get

$$
\Delta_{n-1}(\mathscr{H}, K)=\frac{n}{2} V\left(K, M_{\varphi}, \ldots, M_{\varphi}\right) .
$$

By Minkowski’s inequality,

$$
\Delta_{n-1}(\mathscr{H}, K)^{n} \geq\left(\frac{n}{2}\right)^{n} \lambda_{n}(K) \lambda_{n}\left(M_{\varphi}\right)^{n-1}
$$

with equality if and only if $K$ is homothetic to $M_{\varphi}$, and, by (27) and (31),

$$
\Delta_{0}(\mathscr{H}, K)=\frac{1}{2^{n} \kappa_{n}} \lambda_{n}(K) \lambda_{n}\left(Z_{\varphi}\right) .
$$

This gives

$$
\frac{\Delta_{0}(\mathscr{H}, K)}{\Delta_{n-1}(\mathscr{H}, K)^{n}} \leq \frac{1}{n^{n} \kappa_{n}} \frac{\lambda_{n}\left(Z_{\varphi}\right)}{\lambda_{n}\left(M_{\varphi}\right)^{n-1}}=\frac{\Delta_{0}\left(\mathscr{H}, M_{\varphi}\right)}{\Delta_{n-1}\left(\mathscr{H}, M_{\varphi}\right)^{n}}
$$

with equality if and only if $K$ is homothetic to $M_{\varphi}$. Thus we have the following result.

Theorem 4.3. Let $\mathscr{H}$ be a $\varphi$-uniform hyperplane system in $\mathbb{R}^{n}$, and let $M_{\varphi}$ be the convex body with area measure $\varphi$. Among all convex bodies $K$ for which $\mathscr{X}$ has a given 
$(n-1)$-dimensional number density $\Delta_{n-1}(\mathscr{H}, K)$, precisely the homothets of $M_{\varphi}$ yield the maximal zero-dimensional intersection density $\Delta_{0}(\mathscr{H}, K)$.

In the case of the plane $\mathbb{R}^{2}$ we have $\lambda_{2}\left(Z_{\varphi}\right) / \lambda_{2}\left(M_{\varphi}\right)=4[7$, p. 384] and hence

$$
\Delta_{0}(\mathscr{H}, K) \leq \frac{1}{\pi} \Delta_{1}(\mathscr{H}, K)^{2},
$$

with equality if and only if $K$ is homothetic to $M_{\varphi}$. This yields inequality (7) together with the equality conditions.

It was conjectured in [7] that $\lambda_{n}\left(Z_{\varphi}\right) / \lambda_{n}\left(M_{\varphi}\right)^{n-1} \leq 2^{n}$ for $n \geq 3$, which would imply that

$$
\Delta_{0}(\mathscr{H}, K) \leq \frac{2^{n}}{n^{n} \kappa_{n}} \Delta_{n-1}(\mathscr{H}, K)^{n}
$$

This is still open. More generally, we conjecture that

$$
\Delta_{n-k}(\mathscr{H}, K) \leq \frac{2^{k}}{n^{k} \kappa_{k}}\left(\begin{array}{l}
n \\
k
\end{array}\right) \Delta_{n-1}(\mathscr{H}, K)^{k}
$$

for $k=2, \ldots, n$. The equality sign is attained if $K$ is a cube $Q$ and $\varphi=\varphi_{Q}$ is proportional to the area measure of this cube; in this case the hyperplanes of $\mathscr{H}=\mathscr{H}_{Q}$ that are relevant for the densities are parallel to the facets of the cube. At least, it is true that the most symmetric pair, consisting of an isotropic uniform hyperplane system $\mathscr{H}_{\sigma}$ and the ball, is not extremal, since

$$
\frac{\Delta_{n-k}\left(\mathscr{H}_{\sigma}, B^{n}\right)}{\Delta_{n-1}\left(\mathscr{H}_{\sigma}, B^{n}\right)^{k}}<\frac{\Delta_{n-k}\left(\mathscr{H}_{Q}, Q\right)}{\Delta_{n-1}\left(\mathscr{H}_{Q}, Q\right)^{k}} .
$$

See (10) and the Appendix of [1] and note that the probability $p_{k}(K, \varphi)$ occurring there satisfies

$$
\frac{\Delta_{n-k}(\mathscr{H}, K)}{\Delta_{n-1}(\mathscr{H}, K)^{k}}=\frac{2^{k}}{k ! \kappa_{k}} p_{k}(K, \varphi) .
$$

\section{References}

1. Bauer, Ch., and Schneider, R., Extremal problems for geometric probabilities involving convex bodies. Adv. in Appl. Probab. (to appear).

2. Cohn, D. L., Measure Theory. Birkhäuser, Basel, 1980.

3. Fejes Tóth, L., Lagerungen in der Ebene, auf der Kugel und im Raum. Springer-Verlag, Berlin, 1953.

4. Kuipers, L., and Niederreiter, H., Uniform Distribution of Sequences. Wiley, New York, 1974. 
5. Mecke, J., Inequalities for intersection densities of superpositions of stationary Poisson hyperplane processes. Proc. Second Internat. Workshop on Stereology and Stochastic Geometry (eds. E. B. Jensen and H. J. G. Gundersen), Memoirs, vol. 6, University of Aarhus, 1983, pp. 115-125.

6. Miles, R. E., Poisson flats in Euclidean spaces, Part 1: A finite number of random uniform flats. Adv. in Appl. Probab. 1 (1969), 211-237.

7. Schneider, R., Random hyperplanes meeting a convex body. Z. Wahrsch. Verw. Gebiete 61 (1982), 379-387.

8. Schneider, R., Inequalities for random flats meeting a convex body. J. Appl. Probab. 22 (1985), 710-716.

9. Schneider, R., Convex Bodies: the Brunn-Minkowski Theory. Encyclopedia of Mathematics and Its Applications, vol. 44. Cambridge University Press, Cambridge, 1993.

10. Schneider, R., and Weil, W., Integralgeometrie. Teubner, Stuttgart, 1992.

11. Thomas, C., Extremum properties of the intersection densities of stationary Poisson hyperplane processes. Math. Operationsforsch. Statist. Ser. Statist. 15 (1984), 443-449.

Received January 9, 1994, and in revised form August 15, 1994. 